\title{
Large-gap Peripheral Nerve Repair Using Xenogeneic Transplants in Primates
}

Paul Holzer ( $\sim$ pholzer1@jh.edu )

Johns Hopkins University https://orcid.org/0000-0003-3638-5267

Elizabeth Chang

Xenotherapeutics, Inc. 501(c)3 https://orcid.org/0000-0002-5966-6531

Jamie Tarlton

Glasgow Caledonian University

Diana Lu

Xenotherapeutics, Inc. 501 (c)3

Natasha Gillespie

Glasgow Caledonian University

Kaitlyn Rogers

Xenotherapeutics, Inc. 501 (c)3

Jon Adkins

Xenotherapeutics, Inc. 501 (c)3

Monica Metea

Preclinical Electrophysiology Consulting, LLC

Alan LaRochelle

Biomere Biomedical Research Models, Inc.

Joan Wicks

StageBio

Buket Onel

XenoDiagnostics, LLC

Steve Gullans

Xenotherapeutics, Inc. 501(c)3

Joshua Doloff

Johns Hopkins University https://orcid.org/0000-0002-4323-3264

Linda Scobie

Glasgow Caledonian University

Curt Cetrulo, Jr.

Massachusetts General Hospital

Rod Monroy

Xenotherapeutics, Inc. 501 (c)3 
Article

Keywords: peripheral nerve injuries, neural porcine therapeutics, xenotransplantation

Posted Date: September 3rd, 2021

DOl: https://doi.org/10.21203/rs.3.rs-846240/v1

License: (c) (i) This work is licensed under a Creative Commons Attribution 4.0 International License. Read Full License 


\title{
Large-gap Peripheral Nerve Repair Using Xenogeneic Transplants in Primates
}

\author{
*Paul Holzer ${ }^{1,11}$, Elizabeth J. Chang ${ }^{2,11}$, Jamie Tarlton ${ }^{3}$, Diana Lu ${ }^{11}$, Natasha Gillespie ${ }^{3}$, Kaitlyn \\ Rogers $^{11}$, Jon Adkins ${ }^{11}$, Monica Metea ${ }^{7}$, Alan LaRochelle ${ }^{8}$, Joan Wicks ${ }^{9}$, Buket Onel ${ }^{10}$, Steve \\ Gullans $^{11}$, Joshua Doloff ${ }^{4}$, Linda Scobie ${ }^{3}$, **Curtis L. Cetrulo, Jr. ${ }^{5,6}, * *$ Rod Monroy ${ }^{11}$ \\ ${ }^{1}$ Whiting School of Engineering, Johns Hopkins University, Baltimore, Maryland. \\ ${ }^{2}$ Department of Bioengineering, Northeastern University, Boston Massachusetts ${ }^{3}$ Department \\ of Biological and Biomedical Sciences, Glasgow Caledonian University, Glasgow, UK. \\ ${ }^{4}$ Department of Biomedical Engineering, Translational Tissue Engineering Center, Johns \\ Hopkins University School of Medicine, Baltimore, Maryland ${ }^{5}$ Reconstructive \\ Transplantation Laboratory, Massachusetts General Hospital, Boston, Massachusetts. \\ ${ }^{6}$ Shriners Hospital for Children-Boston, Harvard Medical School, Boston, Massachusetts. \\ ${ }^{7}$ Preclinical Electrophysiology Consulting, LLC, Boston, Massachusetts. ${ }^{8}$ Biomedical \\ Research Models, Inc. (Biomere), Worcester, Massachusetts. ${ }^{9}$ StageBio, Mt Jackson, \\ Virginia. ${ }^{10}$ Xeno Diagnostics, LLC, Indianapolis, Indiana. ${ }^{11}$ XenoTherapeutics Inc., 501(c)3, \\ Boston, Massachusetts. \\ *corresponding author \\ $* *$ co-senior authors
}

\section{Abstract}

\begin{abstract}
Early surgical intervention is required to successfully treat severe, large-gap peripheral nerve injuries. However, all existing treatments have shortcomings, and for large-gap injuries $(\geq 4 \mathrm{~cm})$, there is no reported alternative to autologous nerve. We report preclinical repair of large $(4 \mathrm{~cm})$, complete transectional radial nerve damage in Rhesus macaques using viable, whole sciatic nerve from genetically engineered, designated pathogen free porcine donors. Porcine nerves are physiologically similar to human nerves, contain neurotrophic growth factors and a matrix-rich scaffold, and offer greater clinical availability. We demonstrate regeneration of the transected nerve, distal muscle reinnervation, and recovery of conduction velocity and compound muscle action potential across xenogeneic transplants resulting in functional recovery comparable to autologous controls. We also show the lack of systemic porcine cell migration and the elimination of detectable transplanted porcine tissue. Our findings support the safety and efficacy of neural porcine therapeutics and the broader clinical potential of xenotransplantation.
\end{abstract}




\section{Introduction}

Severe trauma to the extremities frequently results in neurotmesis, the complete transection of peripheral nerves, and these injuries have a devastating impact on patients' quality of life $[1,2]$. Severe peripheral nerve injury (PNI) requires surgical intervention, and it is estimated that twenty million Americans suffer from PNI resulting in nearly 50,000 surgeries annually to repair them [1]. Regeneration of peripheral nerves, even after surgical repair, is slow and often incomplete. Less than half of patients who undergo nerve repair surgery following PNI regain adequate motor or sensory function, and such deficits may result in complete limb paralysis and intractable neuropathic pain [2].

Trauma to the axonal tissue results in an irreversible cascade of apoptosis known as Wallerian degeneration starting within 24-48 hours of injury [3]. Schwann cells phagocytose tissue debris, and macrophages enter the site of injury, further stimulating Schwann cell and fibroblast proliferation via the release of neurotrophic growth factors, such as c-Jun, GDNF, NT-3, and NGF [4]. Up to 100 axonal extensions sprout from the node of Ranvier proximal to the site of injury and mature into a growth cone that elongates toward neurotrophic factors released from the motor and sensory receptors of denervated tissue or the distal nerve stump. If axonal sprouts cannot reach a receptor or endoneurial tube, growth cone branches continue to proliferate stochastically, forming a painful mass of disorganized tissue called a neuroma $[2,5]$.

Thus, optimal surgical therapies should contain a matrix-rich scaffold and neurotrophic growth factors to facilitate this critical axon regeneration process [4-6]. The current standard of care is surgical transplantation of autologous sensory nerve procured from a donor site of the same patient, typically the sural or fibular nerve [6]. Though treatment of motor or mixed-modal nerve bundles with sensory nerves is less than ideal, achieving satisfactory functional recovery in only $\sim 50 \%$ of patients [7], it remains the clinical standard because sacrifice of a motor nerve from the patient represents a clinical and ethical challenge.

Other treatments include allogeneic transplants from human cadaver nerve, biological conduits made from collagen [8], and resorbable nonbiological conduits made from synthetic polymers [9]. However, all clinically available treatments have numerous shortcomings: human autologous and allogeneic transplantation have supply limitations; autologous sensory nerve transplantation is limited by comorbidity and additional surgery may not be feasible in cases of severe injury [5-7]; non-decellularized allogeneic transplantation requires immunosuppressive therapy and risks infectious disease transmission; decellularized allogeneic or nonbiological conduits lack neurotrophic growth factors; and collagen conduits may also lack endoneurial matrix and superstructure $[1,10]$. For injuries spanning $\geq 4 \mathrm{~cm}$ of nerve (large-gap), there are no reported treatment alternatives to autologous nerve transplantion [2]. Thus, there exists a critical, unmet need for high-quality, widely available surgical therapeutics.

We hypothesized that viable, xenogeneic nerve transplants derived from this line of porcine donors may be used for successful reinnervation and recovery of large-gap ( $\geq 4 \mathrm{~cm})$ PNIs, including complete axonal transection (neurotmesis). Viable xenogeneic nerve transplants contain a rich scaffold with neurotrophic growth factors and offer the potential for greater clinical availability without the patient undergoing an additional surgical procedure. Additionally, xenogeneic transplantation allows for the use of mixed-modal nerves containing the endoneurial architecture of motor and sensory fascicle bundles, which may better facilitate 
efferent and afferent conduction through the conduit. Porcine nerves share many physiological characteristics of human motor and sensory nerves, including size, length, extracellular matrix, and architecture [1]. Transplantation from wild type porcine donors suffers from hyperacute immune rejection by the recipient mediated by preformed antibodies against Galactose- $\alpha$-1,3-galactose ( $\alpha$-Gal), an oligosaccharide present on all non-primate mammalian cells, as well as the possibility of zoonotic infectious disease transmission.

Recently, skin xenotransplants derived from genetically engineered $\alpha$-1,3-galactosyltransferase knockout (GalT-KO) designated pathogen free (DPF) porcine donors for human clinical use have demonstrated preclinical [11] and clinical [12] safety and efficacy.

In this study, viable whole sciatic nerve from a GalT-KO porcine donor is used to repair surgically induced $4 \mathrm{~cm}$ radial nerve neurotmesis in ten Rhesus macaques (Macaca mulatta). We compare autologous radial nerve, which is comprised of both motor and sensory nerves, where as the current clinical standard of care is routinely sensory only; therefore, our design represents a more stringent control and comparison between our experimental material. Recipients were split and assessed for morphological, electrophysiological, functional, and immunogenic outcomes over an 8- (Group 1; 1.1 through 1.5) or 12-month (Group 2; 2.6 through 2.10) observational period.

Tacrolimus was administered to both groups for either eight (Group 1) or six (Group 2) months to encourage nerve regeneration, as previously described [13]. At necropsy, we evaluated internal vascular organs, peripheral blood, and other recipient tissues, as well as the transplantation site for histomorphology, toxicology, and the presence of porcine endogenous retrovirus (PERV), a biomarker for xenogeneic cell migration and mixed chimerism. We demonstrate herein the immunologically and microbiologically safe repair of large-gap PNI in a non-human primate (NHP) model using a viable, mixed-modal xenogeneic nerve transplant approach.

\section{Results and Discussion}

\section{Surgical Outcomes}

We selected porcine sciatic nerve tissue as the source of the xenogeneic transplant due to its superstructural similarity to human and primate nerve [1]. The radial nerve was selected as the transplantation recipient site because there are minimal neighboring nerves (Figs. 1a-d). Those in close proximity may reinnervate downstream muscle fibers and complicate electrophysiology and functional analysis of the extensor digitalis muscles. Transplantation at the radial nerve also allowed for ethical loss of function and clearly articulated return of function in an observable and isolated movement. The maximum practical gap size possible was $4 \mathrm{~cm}$ based on the measured lengths of the recipients' limbs. The mean distance from the recipients' proximal neurorrhaphy site to the site of innervation of the extensor carpi radialis longus and extensor carpi radialis brevis muscles [14] measured $15.7 \pm 0.17 \mathrm{~cm}$.

Bilateral, $4 \mathrm{~cm}$ complete transections of radial nerves were surgically introduced in a total of ten Rhesus macaque (NHP) recipients. Two porcine sciatic nerves were harvested from one donor (Figs. 1e, f), trimmed into ten $4 \mathrm{~cm}$ segments and transplanted into one of the radial nerve gaps in each recipient (Fig. 1e), randomized to the left or right limb. In the contralateral arm, excised NHP radial nerve segments were rotated $180^{\circ}$ and reimplanted as a surgical control (Fig. 1e). Surgeries were performed synchronously and the surgical personnel, sterile field, 

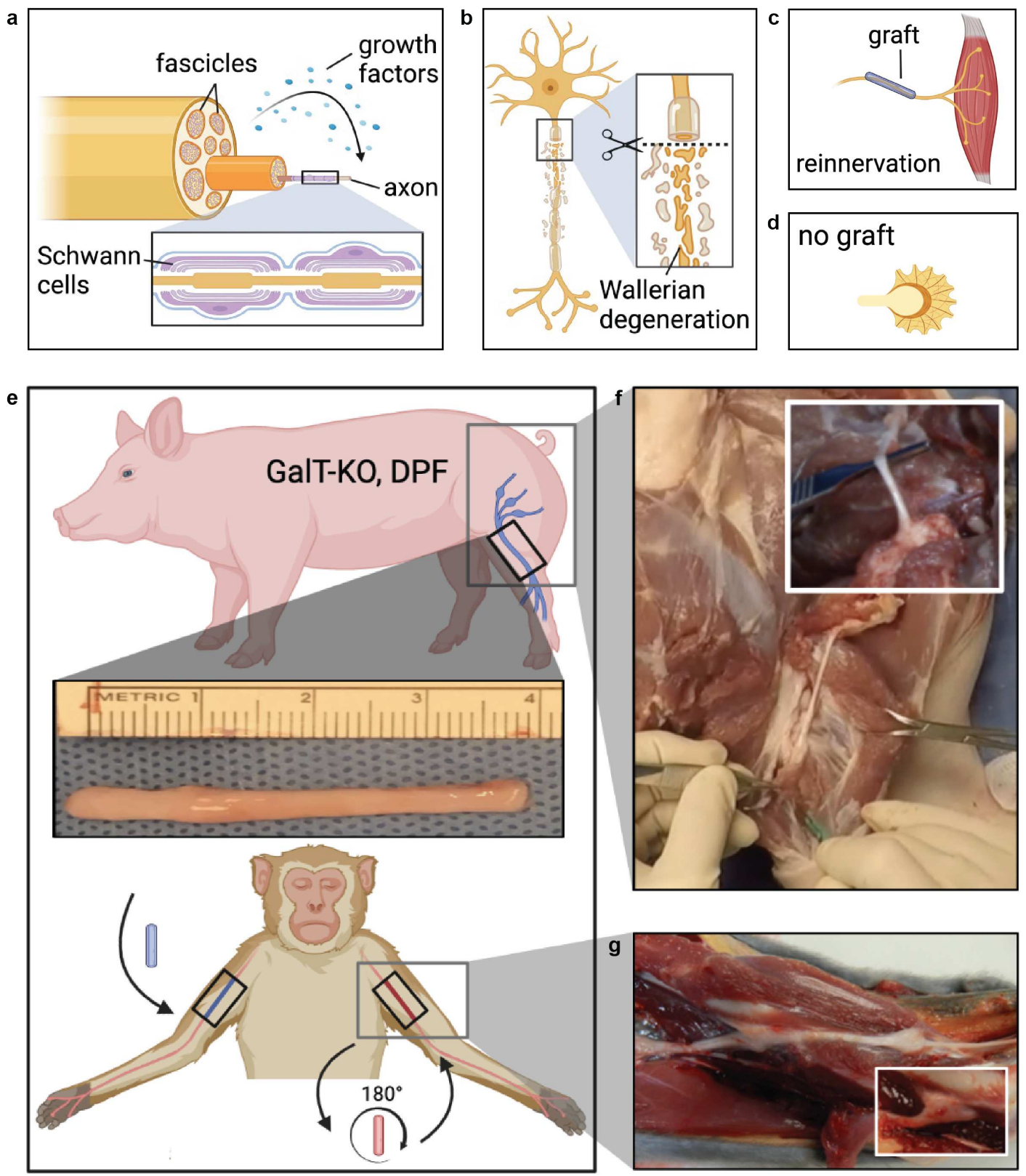

Figure 1. Schematics of peripheral nerve injury (PNI) and study design. a, Peripheral nerve anatomy. b, Transected axons degenerate from the point of injury, moving distally. c, Large-gap nerve injuries require surgical intervention using transplanted conduits between the proximal and distal ends of a cut nerve. d, Without axonal guidance, axonal sprouts will grow stochastically, causing a painful neuroma. e, Schematic of study design. Porcine sciatic nerve is trimmed to $4 \mathrm{~cm}$ and transplanted in primate radial nerve transection, resulting from surgical resection. The transected radial nerve is rotated $180^{\circ}$ and reimplanted as a control. f, Sciatic nerve of porcine donor visualized in situ during procurement surgery. g, Xenogeneic transplant-treated limb at necropsy. A total of ten recipients received both xenogeneic and autologous transplants, one in each arm. Inset shows a neuroma on recipient 2.10. 


\begin{tabular}{|llll|}
\hline \multicolumn{2}{|l}{ Table 1 Histopathology Evaulations Scoring Parameters } \\
Score & $\begin{array}{l}\text { Nerve Bundle } \\
\text { Diameter }(\mu \mathrm{m})\end{array}$ & Myelination & Necrosis/Fibrosis \\
\hline 0 & No nerve & No detectable myelin & None \\
1 & $<100$ & Minimal myelin & Minimal \\
2 & $101-200$ & Moderate myelin & Moderate \\
3 & $201-300$ & Significant myelin & Severe \\
4 & $>300$ & Fully myelinated & Complete \\
\hline
\end{tabular}

surgical technique, and uniformity of the transplant procedure was independently assessed for quality control at each step.

Due to the large number of surgeries, five sciatic nerve segments were transplanted fresh one to two days after donor harvesting while the remaining five were cryopreserved and subsequently thawed for transplantation over two days the week following based on Holzer et. al., 2020 [15]. Cryopreservation of xenogeneic skin transplants has been shown to be safe and effective for up to seven years [16], demonstrating no significant or meaningful differences between fresh and frozen transplants.

All ten subjects tolerated the surgical procedure, there were no systemic adverse events attributed to the xenotransplant, and 19 out of the 20 surgeries were successful. However, recipient 2.10's xenogeneic transplant resulted in a surgical technical failure (Fig. $1 \mathrm{~g}$ inset) and functional non-recovery.. At necropsy, histomorphological analysis revealed a large neuroma proximal to the transplantation site and lack of axonal continuity through and distal to the transplant. Because of this, the recipient's data (both xenogeneic and autologous limbs) were removed from the study resulting in only four recipients in Group 2.

\section{Pathology}

At necropsy, nerve tissue at the bilateral transplantation sites was explanted and examined in a blinded manner. Enlargement of the nerves was observed at the proximal and distal anastomotic sites for both types of transplants for all recipients (Fig. 2a, b). Microscopic examination demonstrated foreign body reaction around the sutures, as well as multidirectional proliferation of small diameter nerve branches consistent with neuroma formation on all transplants, though on a much smaller scale than that of the one surgical failure. This outgrowth and scarring are expected, and successful regeneration is typically observed for only $50 \%$ of the original axons at each coaptation site [2].

Importantly, the response at the anastomotic sites was comparable between the autologous and xenogeneic explants. The response across the tranplanted region consisted of mild fibrosis with embedded nerve fibers coursing mostly longitudinally along the long axis of the transplant.

Explanted tissues were stained by immunohistochemistry for expression of Neurofilament $\mathrm{H}$ to demonstrate axons (Fig. 2c). Nerve bundle diameter for explanted NHP nerves not subsequently used for transplantation (perioperative nerves) were $>300 \mu \mathrm{m}$. For Group 1 
a

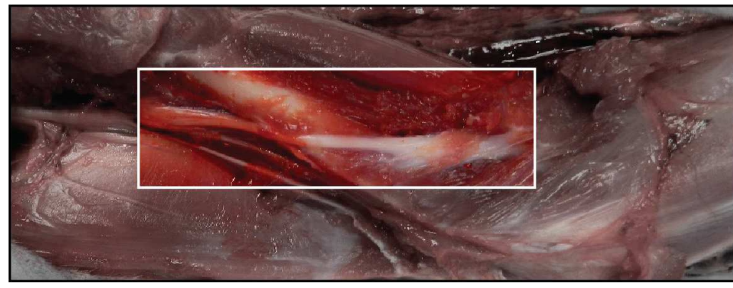

b

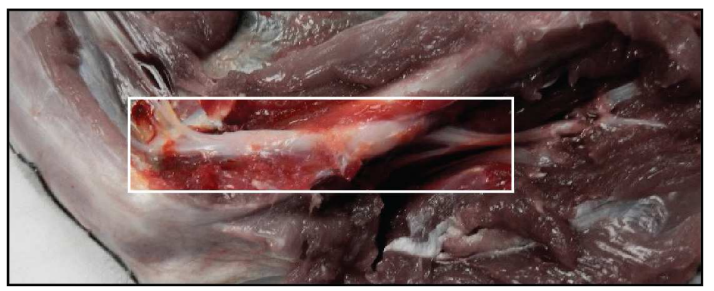

Baseline Autologous Xenogeneic

Group $1 \quad$ Group $1 \quad$ Group 1

Group $2 \quad$ Group 2 $\quad$ Group 2
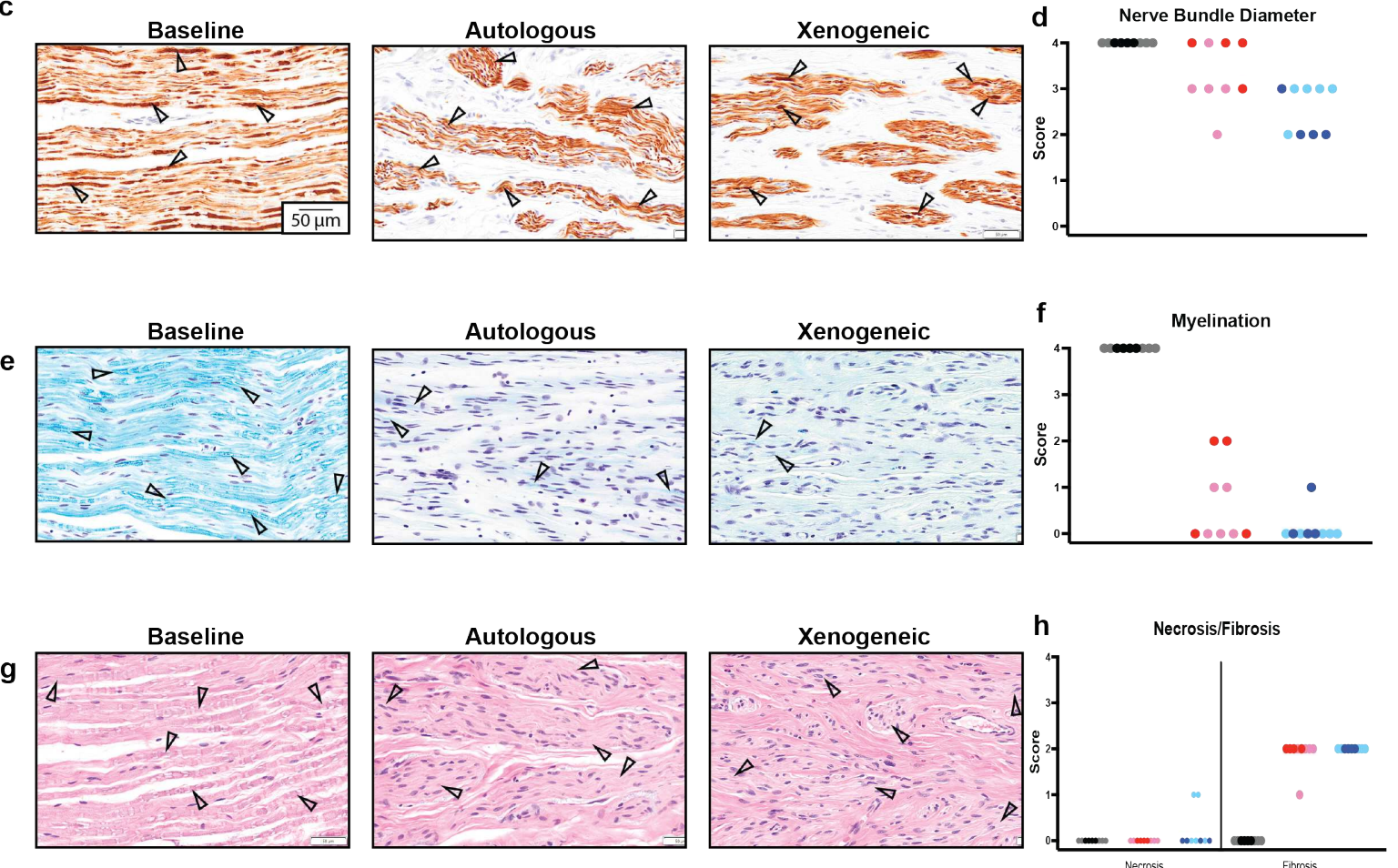

Figure 2. Autologous vs xenogeneic histopathologic analysis of recipients. a, Autologous transplant site (recipient 2.6) after reconstruction and 12-month recovery. b, Xenogeneic nerve transplant of the same recipient. c, Histology sections stained by immunohistochemistry for expression of Neurofilament $\mathrm{H}$. Arrowheads indicate axons. A representative recipient was chosen for each group; 1.1 for baseline, 2.9 for autologous, and 2.6 for xenogeneic for all stains. d, Baseline, autologous, and xenogeneic nerve bundle diameter comparison. Scoring for all histology (c, e, g) based on Table 1. e, Luxol fast blue (LFB) staining was done to score myelination. Arrowheads indicate myelin. f, A myelination drop was observed in surgical groups compared to baseline.g, Necrosis and fibrosis were assessed using H\&E staining. Arrowheads indicate nerve bundles. h, Necrosis and fibrosis were equivalent for autologous and xenogeneic sites. 
(8-month endpoint), the diameter of the transplanted nerve bundles across the defect site for all five recipients were comparable for both types of nerve transplants, ranging from 100 to $300 \mu \mathrm{m}$. At the end of study for Group 2 (12-months), scores for xenogeneic nerve bundle diameters (Table 1) were slightly lower than that of the autologous control, with four autologous controls reaching preoperative diameters. None of the xenogeneic nerve bundles reached preoperative diameters (Fig. 2d) however, they remained comparable in functional recovery. All pathology was scored on a qualitative scale so no statistical analysis was performed.

Luxol Fast Blue staining was used to demonstrate myelination levels of the various regions of the explant (Fig. 2e). Overall, for all recipients and transplants, we observed full myelination in the nerve regions immediately proximal to the transplanted sites and some loss of myelin on the nerve distal to the transplants. No myelination was observed in either xenogeneic or autologous explanted nerves excised at 8-months. Minimal amounts of myelination was observed in one of four xenogeneic explants, and two out of four autologous explants at 12-months, indicating new nerve growth. Representative stains are shown in Figure $2 \mathrm{f}$.

We observed comparable levels of cell death (necrosis) and presence of fibrous connective tissue (fibrosis) in the xenogeneic-transplanted explants compared to that of autologous control (Figs. $2 \mathrm{~g}, \mathrm{~h}$ ). The presence of fibrous tissue indicates tissue damage in the region, and the extent seen in the recipients is consistent with the type of surgical procedure performed. We observed slightly larger nerve fibers and more remyelination in the autologous surgical control, but otherwise there were no meaningful differences in pathology assessments.

Due to fixation (shrinkage) artifact, ideal longitudinal sections were precluded, and adequate cross-sections were not obtained. Possible physiological limitations include variable axon diameter and bundle quantity between the non-human primate and porcine nerves, especially given the use of the sciatic nerve as the transplant source to repair a radial nerve. Importantly, histological evidence indicated that tissue cryopreservation does not negatively impact clinical outcomes.

\section{Functional Evaluation}

Qualitative regain of radial nerve functionality was monitored according to the following categorical scale: no observable impairment (Fig. 3a), mild impairment, moderate impairment, and severe impairment (Fig. 3b). Following surgery, complete loss of radial nerve function was observed bilaterally in all recipients regardless of nerve transplant type used. The rate of recovery averaged across the recipients appeared to be slower in the xenogeneic transplanted limbs for both study groups (Figs. 3c, d), while the overall magnitude of functional recovery was near equivalent between the limbs treated with xenogeneic nerve transplant and the autologous surgical control. Mild impairment was noticed in one autologous and three xenogeneic transplant arms at observational endpoints.

Qualitative functional measurements are inherently limited by subjectivity and the inability to achieve single-degree precision. Electrophysiologica, physical baseline, and behavioral factors may contribute to functional outcomes downstream of successful reinnervation across the transplant. For example, some recipients may be inherently weaker and perform worse due to this. Postoperative physical therapy for recipients would enhance functional outcomes and is widely available for human transplant recipients, but was not performed on NHP recipients in this study. Lastly, some were simply less inclined to reach for the specific food provided 
during reach recordings.

However, even by qualitative categorical rankings, our data suggest that by 8-months postoperative, regain of function was comparable in the xenogeneic transplant and the autologous control, though some xenogeneic limbs experienced delayed functional recovery.

\section{Electrophysiology}

Sensory and motor nerve conduction was evaluated for all nine recipients in both arms at baseline and postoperatively at 5-, 8-, and 12-months [17] using Natus UltraPro with Synergy Electrodiagnostic software [14]. Motor nerve function was assessed across the length of the radial nerve by eliciting orthodromic compound muscle action potentials from the extensor digitorum communis muscle (EDC) via stimulation at four locations proximal and distal to the transplant site. Motor conduction velocity (NCV), compound muscle action potential (CMAP) amplitude and CMAP duration were calculated for each location following the last supramaximal stimulation and then averaged across the nerve sites. Sensory nerve conduction was determined by eliciting sensory antidromic nerve action potentials (SNAPs) directly from the distal branches of the radial nerve as it passes over the extensor pollicis longus tendon. Approximately ten supramaximal stimuli were averaged for each sensory nerve conduction velocity calculation.

An initial decrease in conduction velocity post-surgery is assumed to be due to axonal loss (axonotmesis), and the recovery of conduction velocity suggests reinnervation across the transplants with fast conducting fibers. Following nerve transection, all conduction is expected to drop to near zero within one week [10] if all nerve pathways leading to the recording site were eliminated. At a rate of axonal regeneration [2] of $1 \mathrm{~mm} /$ day, muscle reinnervation was anticipated at approximately 4-months postoperative, and return of conduction was expected to follow. We therefore chose 5-months as our first postoperative timepoint. Recordings for all recipients were performed at month 8, the Group 1 endpoint, and recordings for group 2 were performed at the 12-month study endpoint.

Axillary F-waves were recorded for all recipients at all timepoints, indicating the presence of motor conduction over long neuronal pathways including the proximal spinal segments and the nerve roots. At the first postoperative assessment (5-months), an overall reduction in average motor and sensory conduction velocities $(-38 \%$ and $-53 \%$, respectively) from preoperative values was noted in all transplants: motor $(64.26 \pm 0.33 \mathrm{~m} / \mathrm{s}$ to $39.52 \pm 5.09 \mathrm{~m} / \mathrm{s})$ and sensory $(53.72 \pm 0.06 \mathrm{~m} / \mathrm{s}$ to $25.28 \pm 3.17 \mathrm{~m} / \mathrm{s})$.

By the end of the observational periods (8- and 12-months postoperative), there were no statistically significant or physiologically relevant group differences in motor or sensory conduction velocities between the autologous or xenogeneic reconstructed limbs. At 8-months postoperative, motor conduction had increased to $53.55 \pm 2.15 \mathrm{~m} / \mathrm{s}$ for autologous nerves and $55.85 \pm 1.98 \mathrm{~m} / \mathrm{s}$ for xenogeneic, indicating partial remyelination of fast conducting fibers. At 12-months postoperative, the remaining four recipients demonstrated motor velocities in both autologous and xenogeneic groups recovering to $\sim 97 \%$ of average baseline values (Figs. 3e, f).

In contrast, sensory nerve velocity recovered to $\sim 50 \%$ of baseline levels by 5 -months postoperative and remained at this level until end of study for both groups. This is expected, as the first sprouting fibers of a transected nerve are sensory, followed by significant remodeling 

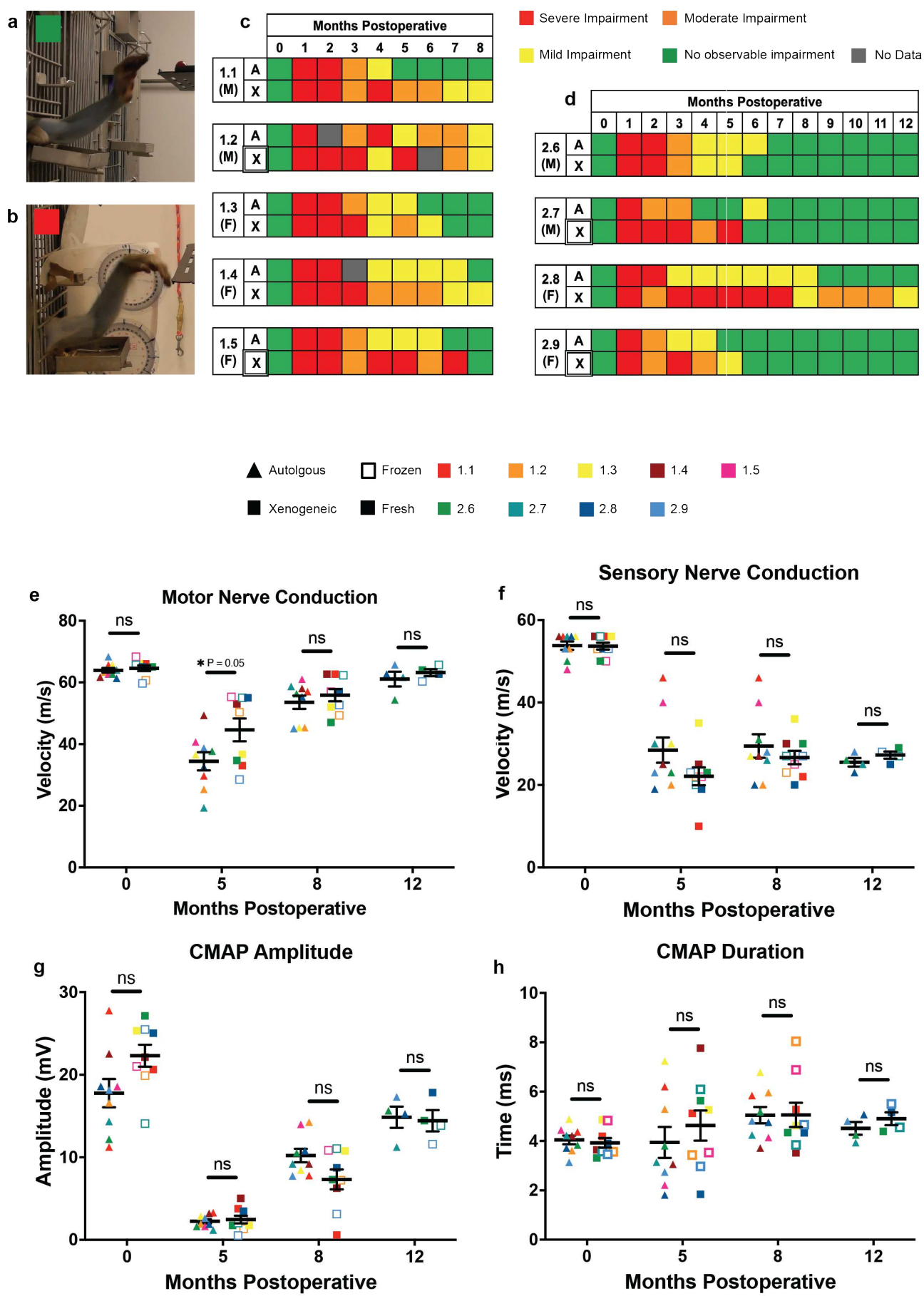

Figure 3. Autologous and xenogeneic transplant recovery was comparable in functional and electrophysiological data. a, Regain of wrist function was qualitatively evaluated based on the amount of impairment observed; a depiction of no observable impairment is shown. b. Depiction of severe impairment. c,d, Groups 1 and recipients' regain of wrist function was qualitatively evaluated and visualized in a heat map. Month 0 indicates baseline values for all graphs and heatmaps. e, Motor nerve conduction velocity showed partial recovery and was similar in both types of implants (*Student's $\mathrm{t}$-Test was used for all comparisons e-h; ns=not significant with a $\mathrm{P} \leq 0.05$ ). f, Sensory nerve conduction velocity showing lack of recovery. g, Compound muscle action potential (CMAP) amplitude showing similar partial recovery between transplant type. $\mathbf{h}$, Compound muscle action potential duration showed similar temporal dispersion between transplant types. 
at the motor unit. However, full recovery of sensory nerve conduction and function has been previously reported to occur over months or years, if at all, and longer observation periods were beyond the scope of this study [2]. Nevertheless, the recovery of any sensory velocity suggests reinnervation of the radial sensory nerves across the transplants.

Average preoperative CMAP amplitudes for all 18 limbs was $20.03 \pm 2.23 \mathrm{mV}$. At 5-months postoperative, a nearly complete loss of action potential was observed in all limbs $(2.52 \pm 0.26$ $\mathrm{mV}$, autologous; $2.47 \pm 0.46 \mathrm{mV}$, xenogeneic), showing that nerves were unable to reach threshold firing levels. By 8-months postoperative, CMAP amplitudes for the autologous nerve transplants had recovered to $10.21 \pm 0.82 \mathrm{mV}$, while limbs treated with xenogeneic nerves recovered to $7.32 \pm 1.20 \mathrm{mV}$. By the end of study, amplitudes for autologous and xenogeneic transplants were equivalent and recovered to $83 \%$ and $65 \%$ of baseline values, respectively (Fig. 3g). The difference in baseline values is not significant, and there were no statistically significant or physiologically relevant differences in CMAP duration between the xenogeneic and autologous transplants at any of the three postoperative timepoints (Fig. 3h). CMAP duration was prolonged in both groups at all timepoints and peaked at 8-months postoperative ( $5.05 \pm 0.33 \mathrm{~ms}$, autologous; $5.06 \pm 0.49 \mathrm{~ms}$, xenogeneic).

The presence of compound muscle action potentials (CMAPs) at the extensor digitalis indicates motor unit reinnervation distal to the transplants. A recovery of nerve conduction velocity indicates the presence of sufficient myelinated, fast-conducting fibers reaching the motor units. Decreased CMAP amplitude and increased duration suggest comparatively fewer axons and myelinated fibers replaced predominantly by thinly myelinated fibers. This was expected, as nerve growth and myelination are relatively slow and progressive processes. We observed no statistically significant or physiologically relevant differences between autologous or xenogeneic treated limbs in the metrics largely indicative of remyelination (CMAP amplitude or duration), consistent with the corresponding histological and functional recovery observations (Figs. 2, 3a-d).

\section{Toxicology}

All recipients received tacrolimus at dosing levels intended primarily to promote nerve regeneration [17], as previously reported [13, 18, 19]. Tacrolimus administration began 14 days before surgery. Postoperative trough levels were maintained below $30 \mathrm{ng} / \mathrm{mL}$ (Fig. 4a), though they varied widely between individual recipients (4.9 to $14.2 \mathrm{ng} / \mathrm{mL}$ ). To determine the effect of discontinuation of tacrolimus on nerve regeneration, the regimen was ceased for five randomly selected recipients at 6-months postoperative (Group 2) and maintained for the remaining five (Group 1) [20].

We observed body weight loss during the tacrolimus administration phase for several recipients in both groups. Following cessation of tacrolimus at 6-months postoperative, we observed gradual, continued weight increase in Group 2 recipients. In contrast, by 8-months on the regimen, Group 1 presented with progressing symptoms associated with tacrolimus toxicity [20] such as limited mobility in knee joints, muscle rigidity, stiffness, and atrophy, as well as significant body weight loss in one recipient. As a result, these five recipients were euthanized [11], and the remaining five recipients (Group 2) survived without incident to the 12-month end of study.

At necropsy samples of the spleen, liver, kidney, and other organs were collected (Fig. 4b). There were no abnormal veterinary observations at necropsy, or on histopathologic evaluation 
of internal organs, peripheral blood, and other systemic tissues for all recipients, regardless of treatment. Hematology and chemistry analysis similarly demonstrated no abnormal findings $[21,22]$.

Red blood cell (RBC), white blood cell (WBC), and platelet (PLT) counts were within normal ranges. Individual WBC types and their percentage of overall WBC, Hemoglobin (HGB), mean corpuscular volume (MCV), hematocrit (HCT), urea nitrogen (BUN), creatinine (CREA), and electrolyte levels were stable at expected baseline levels for the duration of the study[21, 22]. Neutrophil and lymphocyte percentages varied month-to-month, but absolute counts remained close to expected ranges $(2.40 \pm 6.18 \mathrm{~K} / \mu \mathrm{L}$ and $2.46 \pm 8.94 \mathrm{~K} / \mu \mathrm{L}$ respectively) [21]. Glucose (GLU) levels were above expected values [22] and were elevated for all months except zero and twelve (Figs. 4c-g). We expect this was due to the recipients' increased sugar consumption during radial nerve functional evaluations.

In all recipients, xenogeneic transplants caused no adverse events or systemic effects. Tacrolimus toxicity exhibited by long-term administration limited the study's potential analysis and statistical power. Other limitations include the lack of a non-tacrolimus-treated control group necessary to elucidate the relative benefit of the regimen and the lack of baseline hematology and clinical chemistry assessments prior to tacrolimus administration.

\section{Immunogenicity}

Recipients were iteratively assessed for anti-porcine IgM and IgG antibodies to evaluate their response to peripheral blood mononuclear cells (PBMCs) from GalT-KO porcine donors, and for total circulating IgM and IgG levels to assess changes in systemic immunogenicity over the course of the study (Figs. 5a-d). These are measurements of circulating IgM and IgG levels in each recipient, therefore each data point in Figures 5a-d represents one recipient NHP, not the xenogeneic or autologous limb. Importantly, tacrolimus withdrawal at month six did not increase Group 2 total or anti-porcine $\operatorname{IgM}$ or IgG.

Binding of anti-porcine IgM and IgG was assessed using Median Fluorescence Intensity (MFI) and relative MFI obtained as follows: Relative MFI = Actual MFI value / Limit of Blank (MFI obtained using secondary antibody only in the absence of serum). Anti-porcine IgM and IgG levels (Figs. 5a, b) consistently showed an increase in anti-xenogeneic IgM and $\operatorname{IgG}$ above pre-existing levels followed by a gradual decrease to baseline over time. The highest IgM fold increases were detected for all recipients at one week ( 0.25 months) and month three, and the highest IgG fold increases were detected for all recipients in month one and three. These trends were consistent with our previous experience [11] and ongoing clinical trial [12].

Mean anti-porcine $\operatorname{IgG}$ was significantly higher in Group 2 than Group 1 in months one through eight $(\mathrm{P}=0.04)$ (Fig. 5b). The highest level of total IgM and IgG was observed 1-month postoperative in recipient 2.2. We attribute this to inherent immunological differences between recipients, which may also have contributed to the observed inflammation, immune cell infiltrates, and tertiary lymphoid nodules (Fig. 5e) present in the transplant sites. These factors may also contribute to a subtle impact on overall axonal regeneration and functional recovery [23].

Total serum IgM and IgG (Figs. 5c, d) were measured using a commercial ELISA. Total IgM levels were slightly elevated above preoperative levels at one or more postoperative timepoints in all recipients. Likewise, total IgG levels were slightly increased above preoperative levels on sampling months post-transplantation. Overall, the changes detected in total serum IgM 
a

Tacrolimus vs Weight

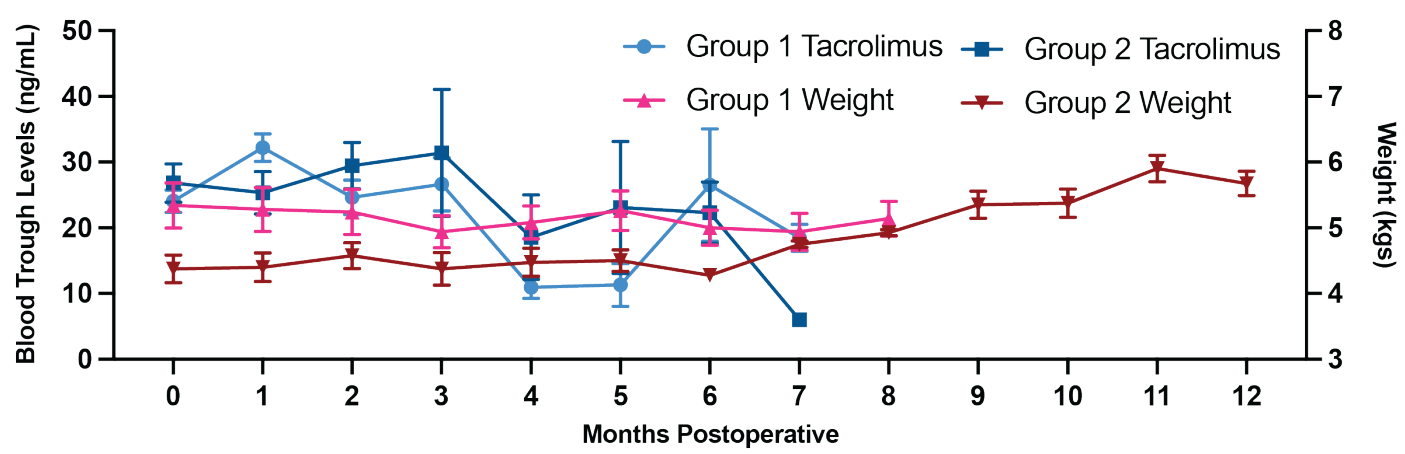

b
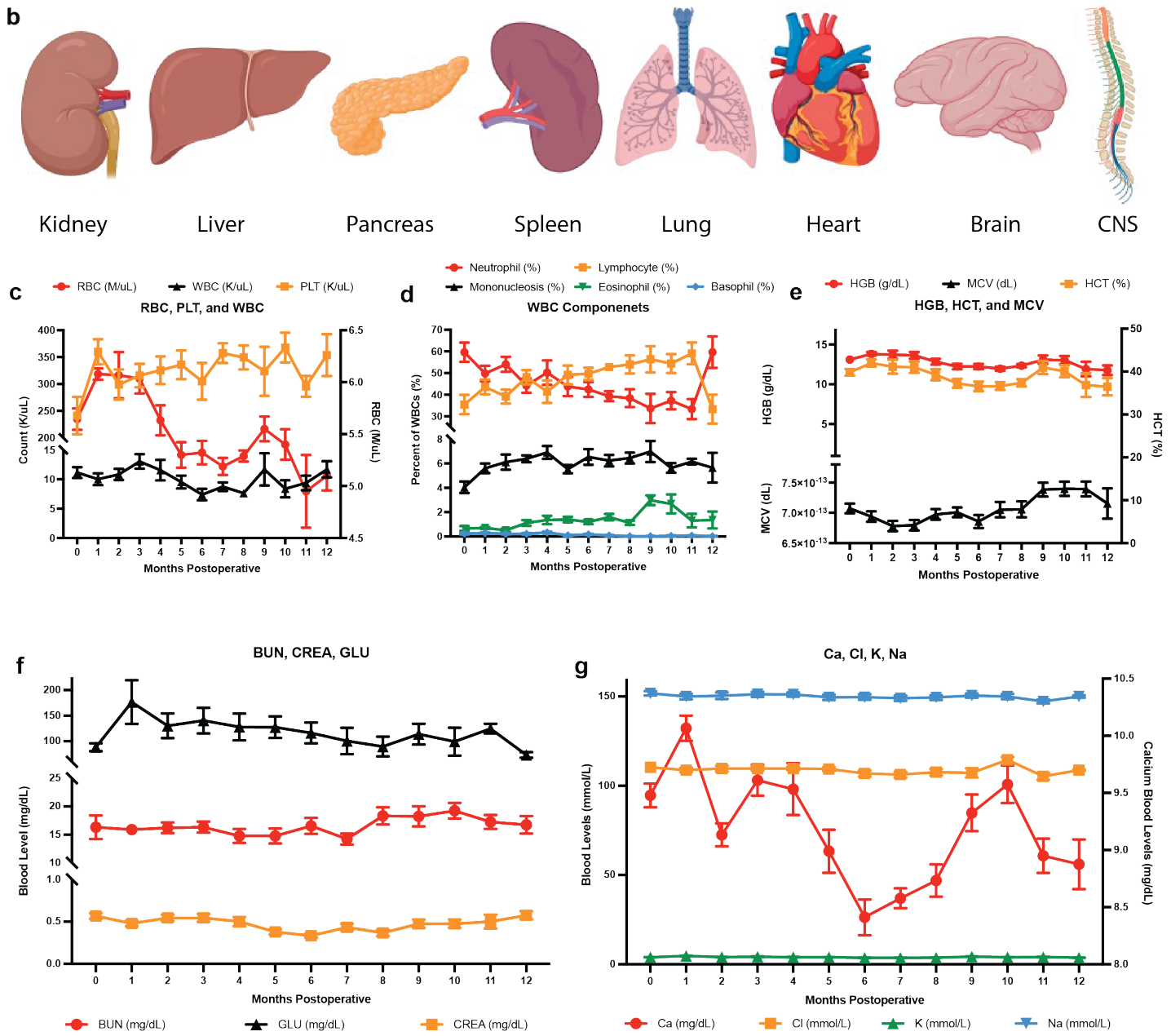

Figure 4. Safety and systemic tolerability of xenogeneic nerve transplantation. a, Body weight loss was observed during the tacrolimus administration phase for several recipients. Month 0 indicates baseline values for all graphs. b, At necropsy samples of various organs were collected. All microscopic findings were considered incidental. c, Red blood cell (RBC), white blood cell (WBC) and platelet (PLT) counts were within normal limits. d, Individual WBC types remained at expected values for the entirety of the study. e, Hemoglobin (HGB), mean corpuscular volume (MCV), and hematocrit (HCT) remained near baseline throughout the study. f, Blood urea nitrogen (BUN) and creatinine (CREA) levels were within normal limits throughout the study. Glucose (GLU) levels were well above expected values maximum of $96.95 \mathrm{mg} / \mathrm{dL}$ with elevated values compared to baseline for all months except 0 (baseline) and 12. g, Electrolyte levels were stable and close to expected baseline values. 
and IgG levels did not exceed increases or decreases greater than $20 \%$ of baseline levels in each of the recipients in Groups 1 and 2 and remained stable over the course of the 12-month study.

There was a notable difference in the infiltration of inflammatory cells within the transplants. By the 8-month timepoint, the overall inflammation for the autologous transplant sites was observed to be minimal (scattered lymphocytes and macrophages), with one lymphoid follicle apparent in one site. In contrast, the xenogeneic transplantation sites had minimal to moderate inflammation: lymphocytes and macrophages with prominent lymphoid follicles (Fig. $5 e)$.

The greatest difference between the xenogeneic and autologous transplantation sites was the presence of tertiary lymphoid nodules in the xenogeneic transplantation sites. Tertiary nodules are organized lymphoid follicles with a center comprising macrophages and dendritic cells. Lymphocytes in the surrounding tissue are indicative of a low level, long-term immune response consistent with porcine tissue clearance and nerve remodeling observed at the transplantation site [24].

\section{Biodistribution of Porcine Tissue}

PERV copy number and expression were analyzed by qPCR to assess the presence of PERV DNA and mixed chimerism. Samples analyzed included xenogeneic and autologous nerve tissues harvested at 8- and 12-months postoperative, sera and PBMCs from the nine subjects obtained at various timepoints over the 12-month study, and spleen, kidney, liver, heart, and lung samples obtained at necropsy.

Evidence of circulating porcine DNA in PBMCs, blood, and tissues analyzed was absent, confirming no porcine tissue was present beyond the transplantation site and no active replication nor incorporation into primate DNA had taken place. Likewise, the autologous transplantation site lacked the presence of PERV or porcine cells.

Surprisingly, the xenogeneic sites were also negative for the presence of PERV/mixed chimerism by qPCR suggesting no residual porcine tissue in the xenogeneic nerve tissue tested. Previous testing of skin xenotransplants demonstrated that porcine cells were detectable at the graft site [15]. In this study, qPCR for the porcine centromere was negative. To confirm whether porcine or primate tissue was present, qualitative PCR using a primate specific target gene demonstrated the presence of primate cells in both autologous and xenogeneic transplants (Fig. 5f). All samples were positive for either the internal positive control (sera) or the control reference genes indicating the validity of the analysis (data not shown).

Recently, it has been suggested that detection of PERV DNA may also be indicative of an inflammatory reaction in addition to testing for infection [25]. Currently, NHPs are not considered a suitable model for PERV infection [26]. We therefore used PERV as a biomarker for presence of porcine cells.

\section{Conclusion}

We performed comprehensive assessments of multiple parameters over 12 months to observe the repair of large-gap PNI treated with xenogeneic transplantation therapeutic. Taken together, 

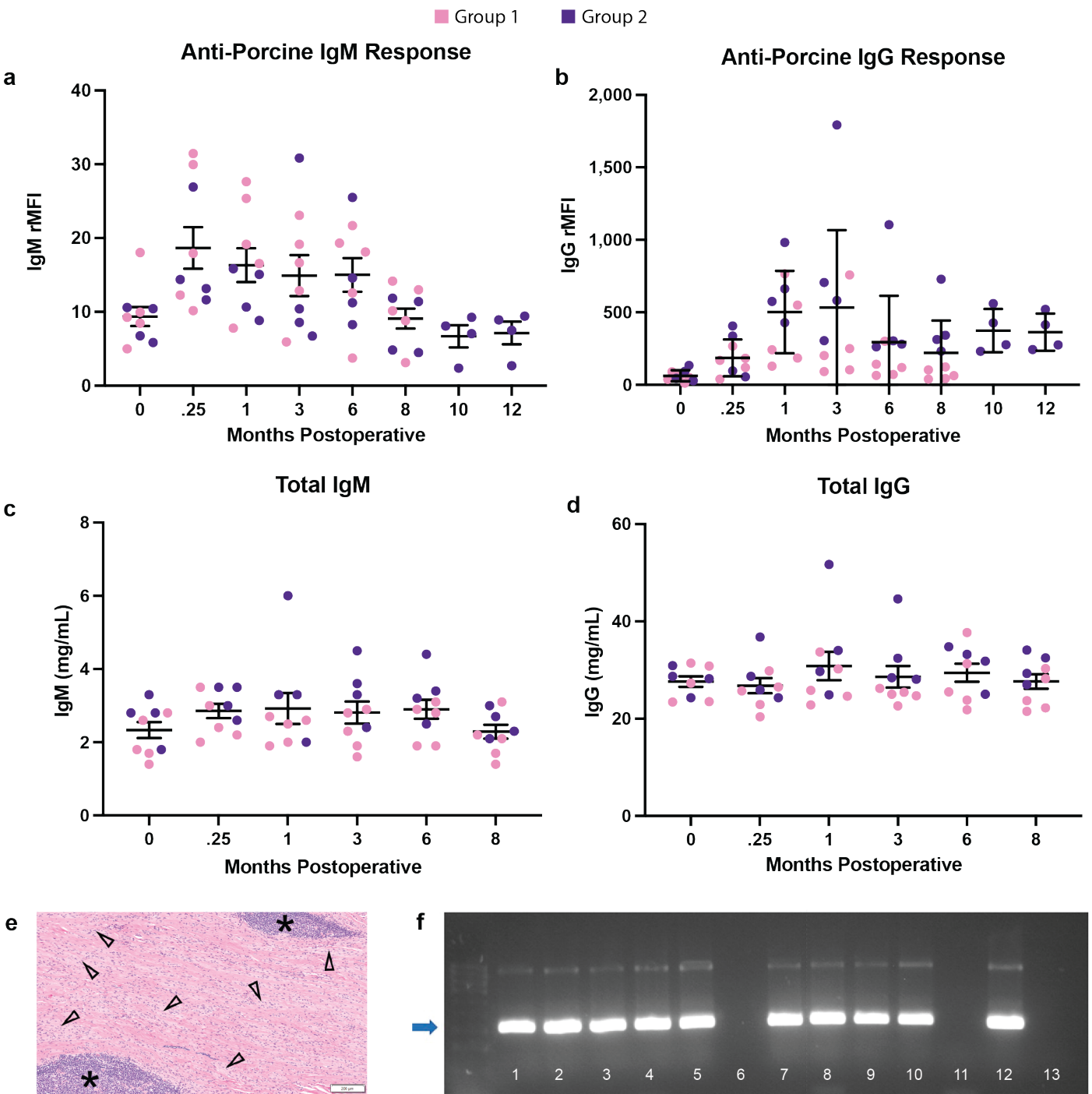

Figure 5. Immune tolerability and complete remodeling of xenogeneic transplanted nerve. a, Anti-Porcine IgM response from baseline (month 0 ) to end of study ( 8 or 12 months). Each point represents an individual recipient's data. b, Anti-Porcine IgG response from baseline to the end of study. c, Total IgM serum levels from baseline to month 8. d, Total IgG serum from baseline to month 8. e, Histology section of 2-6's xenogeneic site explant stained with H\&E. Asterisks indicate lymphoid follicles. Arrowheads indicate nerve bundles. f, Primate-specific PCR on autologous (lanes 2-6) and xenogeneic (lanes 8-12) transplant sites. Lane 13 is the negative control, lane 14 is primate control, lane 15 is porcine control. Qualitative PCR showed primate DNA in both autologous and xenogeneic transplant sites. 
these data suggest a mechanism of peripheral nerve regrowth through the conduit provided by the xenogeneic transplant and the gradual immune clearance of porcine cellular material. The new NHP nerve reinnervated the extensor digitalis, allowing functional recovery of the transected limb. Overall, the functional and morphological properties of the repaired nerves were comparable using xenogeneic or autologous transplant material.

Importantly, we chose to compare our xenogeneic treatment method to a mixed-modal surgical control that is more stringent than the current standard of care. All surgeries were performed consistently, with the same surgical technique, sterile field, suturing, personnel, and transplantation techniques. This allows for discrete control over any possible variance that may arise in both donor and recipient surgical procedures. Though we only used one biological replicate of porcine donor, we expect consistent results in future use of transplant material from a genetically- and age-matched porcine population to harvest nerves with comparable morphology and physiology.

Our long-term, in vivo data suggest promising safety and tolerability following PNI repair with viable porcine nerve transplants. In particular, the lack of systemic porcine cell migration over 12-months in recipients and elimination of detectable transplanted porcine tissue is consistent with complete remodeling of and reinnervation through the transplanted nerve. Discontinuation of tacrolimus at month 6 was not observed to be deleterious systemically and did not impact transplant immunogenicity and functional recovery. These data are encouraging and warrant further evaluations of PNI xenotransplantation with and without tacrolimus treatment.

Results of motor nerve action potential and qualitative functional evaluation suggest downstream muscle reinnervation and functional recovery. Combined, these data warrant further clinical and mechanistic evaluation of porcine xenogeneic transplantation for the treatment of large-gap PNIs. Our results are encouraging for neural porcine xenotransplantation therapies and more broadly support the clinical potential of the field of xenotransplantation.

\section{Methods}

\section{Animals}

This study's surgical procedures, protocols, and guidelines for animal care were independently IACUC reviewed and monitored, and were conducted in accordance with US-FDA 21 CFR Part 58.351 and GFI 197, USDA Animal Welfare Act (9 CFR Parts 1, 2, and 3), the Guide for the Care and Use of Laboratory Animals. All xenogeneic nerve transplants used in this study were sourced from one genetically engineered alpha-1,3-galactosyltransferase knock-out (GalT-KO), designated pathogen free (DPF) porcine donor11. Five male and five female naïve rhesus macaques (Macaca mulatta) served as xenogeneic nerve transplant recipients.

\section{Surgical Procedures}

The porcine donor was euthanized and prepared for surgery as previously described [12]. To isolate the sciatic nerve prior to harvesting, a linear incision was made midway between the sacrum and the ischium and extended ventrally along the posterior aspect of the femur, longitudinally dissecting the gluteus medius, gluteus maximus, piriformis, and biceps femoris muscles, to the proximal tibiofibular joint (Fig. 1). The sciatic nerve was visualized and 
harvested by radial transections distal to the nerve origin and proximal to the bifurcation into the tibial and common peroneal nerves. This process was repeated on the bilateral side. One unmodified sciatic nerve segment was stored in RPMI media and maintained at $4^{\circ} \mathrm{C}$ until surgical use 48 hours later. The other was cryopreserved [27] and stored at $-80{ }^{\circ} \mathrm{C}$ for one week.

Prior to transplantation, xenogeneic nerves were trimmed to $4 \mathrm{~cm}$ to fit the defect size. Large-gap $(\geq 4 \mathrm{~cm})$ peripheral nerve defects were surgically introduced bilaterally in all ten NHP recipients. Recipients, under anesthesia [10], were positioned in lateral recumbency with the shoulder at $90^{\circ}$ flexion, full internal rotation, and neutral abduction. The subcutaneous tissue and deep fascia were dissected for anatomical orientation. A 6-8 cm skin incision was made along the posterolateral margin of the proximal arm towards the antecubital fossa. This procedure exposed the long and lateral heads of the triceps, which converge to form the triceps aponeurosis [28]. The intramuscular plane between the long and lateral head of the triceps was developed approximately $2.5 \mathrm{~cm}$ proximal to the apex of the aponeurosis where the radial nerve and accompanying vessels were observed against the humerus in the radial groove. The surgical plane was extended proximally and distally to minimize unintended injury. The radial nerve was distally transected approximately $1 \mathrm{~cm}$ proximal to the origin of the deep branch. A $4 \mathrm{~cm}$ segment was removed to create the defect and saved for reattachment or subsequent analysis.

Nerve transplants were attached proximally and distally with four to eight equidistant 8-0 nylon monofilament sutures at each neurorrhaphy site. The incision was then closed in layers using subcuticular, absorbable sutures. This process was performed bilaterally per each of the ten recipients; both xenogeneic and autologous nerves were transplanted in the same surgical procedure. Limb designation (right/left) for xenogeneic or autologous transplants was randomly assigned and blinded from observers for analysis. The nine recipients were randomly, evenly divided between two surgical series, one week apart. Five fresh xenogeneic transplants were used in the first series, and five thawed, previously frozen viable porcine xenogeneic transplants were used in the second.

\section{Pathology}

Animals terminated at the scheduled 8- or 12-month time points underwent comprehensive necropsy. Explants of the entire autologous or xenogeneic transplant, including proximal and distal nerve, as well as samples of spleen, liver, kidney, lung, and heart were collected, fixed in $10 \%$ neutral buffered formalin, and transferred to $70 \%$ ethanol after approximately 72 hours. Nerve explants were trimmed longitudinally, routinely processed and embedded in paraffin blocks. Resulting blocks were sectioned, and stained with either hematoxylin and eosin (H\&E), Luxol Fast Blue (LFB), or immunohistochemically stained for neurofilament $\mathrm{H}$ (NF-H). Spleen, liver, kidney and heart were trimmed, processed, embedded in paraffin, sectioned and stained with H\&E. All tissues were evaluated in a manner blinded to treatment. Nerve explants were evaluated for morphologic changes and underwent semi-quantitative scoring according to the criteria in Table 1. All measurements of axon diameter were made by the pathologist using an ocular micrometer. 


\section{Electrophysiology}

Evaluations [17] and analysis were performed using a Natus Neurology System. All motor and sensory responses were elicited with a pediatric stimulator and subcutaneous needle electrodes were used for recordings. Recording procedures were adapted from routinely used neurological clinical protocols. Motor nerve recordings were performed orthodromically at the extensor digitorum communis (EDC) muscle, with successive orthodromic stimulations in the antecubital fossa, at the spiral groove, across the transplant, and at the axilla between the coracobrachialis and the long head of the biceps. Sensory nerve action potentials were recorded antidromically from the radial sensory branch over the extensor pollicis longus tendon with stimulation over the radial side of the forearm, $\sim 5 \mathrm{~cm}$ proximal. The motor nerve conduction velocity (NCV) for each segment was calculated using the differences in onset latency and distance between each two points of stimulation along the radial nerve following supramaximal stimulation. Values were averaged to provide an overall mean of each recipient's motor NCV per arm. CMAP amplitude was determined at the peak of the response following supramaximal stimulation of the associated nerve and the CMAP duration marked at the repolarization. Segmental conduction velocities across the radial nerve were averaged. Sensory nerve responses from approximately ten supramaximal stimuli were averaged at each timepoint. The conduction velocity was calculated using the onset latency of the response and the distance (for sensory $\mathrm{NCV}$ ) or the distance difference (for motor $\mathrm{NCV}$ ) from the stimulation cathode to the recording site.

\section{Functional Evaluation}

A previously reported radial nerve injury mode [29] was adapted to assess the functional recovery of xenogeneic and autologous nerve transplant recipients. Radial nerve injury proximal to the elbow results in a loss of wrist extension function, or "wrist drop," loss of forearm muscle tonality, and digital extension due to motor denervation of the extensor carpi radialis longus and extensor carpi radialis brevis muscles [30, 31]. Radial nerve functional assessments were performed monthly for each recipient and included chair and cage-side observations of active and passive wrist angle flexion during the recipient's retrieval of objects requiring wrist angle extension to obtain them. A series of wrist extension and gripping attempts by each recipient were video recorded, for each isolated arm, for each month. Observations were performed using food treats or mechanical stimulation to encourage wrist extension and gripping. This resulted in 16:13:33 hours of data, for 18 limbs of nine NHP transplant recipients. Over the entire study period a combined 2,057 total events were recorded. Results were analyzed by two independent investigators in a blinded manner with respect to the transplant type and location.

\section{Immunogenicity}

Binding of xenoreactive antibody to pig cells was measured as previously described [32]. Cryopreserved genetically modified alpha-1,3-galactosyltransferase knockout (GalT-KO) porcine PBMCs were thawed and cell concentration was determined using Coulter MD II (Coulter Corporation, Miami, FL). Cells were diluted to a concentration of $1.5 \times 106 \mathrm{cells} / \mathrm{mL}$ in FACS buffer (1X Hanks' Balanced Salt Solution (HBSS) with calcium and magnesium, $0.1 \%$ BSA, and $0.1 \%$ sodium azide). Decomplementation of the serum samples was carried out by heat inactivation for $30 \mathrm{~min}$ at $56{ }^{\circ} \mathrm{C}$ and diluted at 1:2,10,100,1,000, and 10,000 ratios using FACS buffer. $100 \mu \mathrm{L}$ of the cells were added into each well in 96 -well u-bottom 
plate with $10 \mu \mathrm{L}$ of the diluted serum samples and incubated for $30 \mathrm{~min}$ at $4{ }^{\circ} \mathrm{C}$. Cells were washed one time using $200 \mu \mathrm{L}$ FACS buffer. To prevent nonspecific binding, cells were incubated in $100 \mu \mathrm{L} 10 \%$ goat serum for $10 \mathrm{~min}$ at room temperature followed by one more additional washing. Cells were stained with goat anti-human IgG PE and goat anti-human IgM FITC (Jackson ImmunoResearch Laboratories Inc., West Grove, PA) for 30 min at $4{ }^{\circ} \mathrm{C}$. Cells were washed two times using FACS buffer and resuspended in $200 \mu \mathrm{L} 0.5 \%$ PFA in MACS/1X PBS buffer. Flow cytometric analysis completed on Novocyte flow cytometer (ACEA Biosciences, Inc. San Diego, CA). Flow cytometry data was analyzed using NovoExpress 1.3.0 (ACEA Biosciences, Inc.).

Binding of IgM and IgG was assessed using relative mean fluorescence intensity (MFI): Relative Actual MFI value/MFI obtained using secondary antibody in the absence of serum. IgG and IgM ELISA kits from Life Diagnostics were used for the quantification of total circulating IgG and IgM in rhesus serum by following the manufacturer's instructions.

\section{PCR}

Twenty milligrams of the xenogeneic porcine tissue samples and $7 \mathrm{mg}$ of autologous primate tissue samples were treated with the DNeasy Blood and Tissue Kit (Qiagen, Crawley, UK) as described by the manufacturer that included the RNase A-treatment step. The isolated DNA was quantified by UV spectrophotometry. Quantitative PCR amplification of $18 \mathrm{~S}$ (Eurogentec, Seraing, Belgium) was caried out to assess the DNA homogeneity across samples. Serum samples were processed using the Viral RNA mini kit (Qiagen, Crawey, UK) as described by the manufacturer incorporating the DNA digestion step using DNase I to isolate viral RNA. Samples were then processed using the RNeasy MinElute Cleanup kit (Qiagen, Crawley, UK). All Serum samples shown to have an IPC CT $<32$ progressed to PERV transcription analysis. PMBC samples were processed for DNA isolation using a modified version of the manufacturers "Whole Blood" protocol for the Gentra Puregene Blood kit (Qiagen, Crawley, UK). The modified protocol involved homogenizing the PBMC samples prior to RNase A treatment, protein precipitation and finally isopropanol and $70 \%$ ethanol washes were added before DNA hydration. The DNA product was quantified using UV spectrophotometry and $18 \mathrm{~S}$ amplification carried out to assess DNA homogeneity between samples while using $200 \mathrm{ng} /$ reaction. PBMC samples shown to have an $18 \mathrm{~S} \mathrm{CT}<27$ progressed to PERV copy number and mixed chimerism analysis. At necropsy, tissue samples of Kidney, Liver, Lung and Spleen were harvested. RNA isolation was conducted on $\sim 35 \mathrm{mg}$ of the tissue samples using the RNeasy mini kit (Qiagen, Crawley, UK) with homogenization using a Fast-Prep 24 (MP Biomedicals, Eschwege, Germany). The RNA product was quantified using UV spectrophotometry and $18 \mathrm{~S}$ amplification carried out to assess DNA homogeneity between samples while using $200 \mathrm{ng} /$ reaction. Tissue samples shown to have an $18 \mathrm{~S}$ CT $<13$ progressed to amplification, reverse transcription, and PERV copy number and mixed chimerism analysis.

Amplification was carried out using an Applied Biosystems ViiA 7 Real-Time PCR System with a polymerase activation step $\left(10 \mathrm{~min}\right.$ at $\left.95^{\circ} \mathrm{C}\right)$ and 40 amplification cycles of $15 \mathrm{sec}$ at $95^{\circ} \mathrm{C}, 30$ sec at $53{ }^{\circ} \mathrm{C}$, and $30 \mathrm{sec}$ at $60^{\circ} \mathrm{C}$. All primate and porcine transplantation site samples shown to have an $18 \mathrm{~S} \mathrm{CT}<29$ progressed to PERV copy number and mixed chimerism analysis. PERV genome copy number quantification and mixed chimerism was assessed by quantitative PCR (qPCR) using the QuantiTect virus kit (Qiagen, Crawley, UK), with identical cycling conditions described above. PERV was assessed using TaqMan primers specific to the PERV-pol gene as 
previously described [33]. PERV quantification was carried out by comparison to standards of known PERV copy number. The limit of quantification (LOQ) for PERV using this assay is ten copies per reaction. Mixed chimerism was assessed using TaqMan primers for porcine centromeric DNA that were also used in the study described [33]. Detection of porcine cells was quantified by comparison to standards of known porcine cell content. The LOQ for porcine cells using this assay is 0.026 cells per reaction.

Qualitative PCR for the reference gene RPL13A (ribosomal protein gene) was carried out to confirm DNA was suitable for amplification. Primers used were 5'-CCT GGA GGA GAA GAG GAA AGA GA-3' and 5'-TTG AGG ACC TCT GTG TAT TTG TCA AG-3' giving an amplicon of $126 \mathrm{bp}$. Fifty nanograms of DNA and $0.5 \mu \mathrm{M}$ primers in a total volume of $25 \mu 1$ was cycled under the following conditions; $5 \mathrm{~min}$ at $94{ }^{\circ} \mathrm{C}, 50 \mathrm{x}\left(10 \mathrm{sec}\right.$ at $94{ }^{\circ} \mathrm{C} ; 15 \mathrm{sec}$ at $58^{\circ} \mathrm{C}$; $15 \mathrm{sec}$ at $\left.72{ }^{\circ} \mathrm{C}\right) 10 \mathrm{~min}$ at $72{ }^{\circ} \mathrm{C}$. This PCR will detect both primate and porcine material.

Detection of primate specific DNA was carried out using qualitative PCR as described previously [34] with modifications. In brief, primers used were P5 5'-ATC TGG ACC AGA AAT CCC GAC GAT ATT ACT AAT GAG GAG-3' and P6 5'-CTT GTA GTT CTC TTT ATC TTC CGC CAG TTC AGT AAA GAG-3' giving an amplicon of $450 \mathrm{bp}$. Using the Taq PCR core kit (Quiagen, Surrey, UK) $75 \mathrm{ng}$ of DNA and $0.2 \mu \mathrm{M}$ primers in a total volume of $50 \mu \mathrm{l}$ was cycled under the following conditions; $94^{\circ} \mathrm{C}$ for $5 \mathrm{~min}, 40 \mathrm{x}\left(30 \mathrm{sec}\right.$ at $94{ }^{\circ} \mathrm{C}, 30 \mathrm{sec}$ at $5{ }^{\circ} \mathrm{C}$, and $60 \mathrm{sec}$ at $\left.72{ }^{\circ} \mathrm{C}\right), 10 \mathrm{~min}$ at $72{ }^{\circ} \mathrm{C}$.

\section{Hematology and Clinical Chemistry}

Tacrolimus administration was initiated 5-14 days before surgical procedures via intramuscular injection in the ten rhesus monkey recipients. Postoperatively, all recipients received tacrolimus for at least six months [17] and trough levels were maintained below $30 \mathrm{ng} / \mathrm{mL}$.

Serum chemistry blood samples were processed for serum or transferred to the Biomere Testing Facility laboratory and processed. Whole blood hematology samples were transferred to the Testing Facility laboratory without processing. Whole blood was analyzed on an IDEXX Procyte analyzer for erythrocyte count, hemoglobin, hematocrit, platelet count, leukocyte count, reticulocyte count, and mean corpuscular volume, hemoglobin and hemoglobin concentration, Serum samples were analyzed using an IDEXX Catalyst analyzer (Chem15, Lyte4, Trig, and AST slides for A/G ratio, alanine aminotransferase, albumin, alkaline phosphatase, aspartate aminotransferase, calcium, chloride, cholesterol, creatinine, gamma glutamyl transferase, globulin (by calculation), glucose, inorganic phosphate, potassium, sodium, total bilirubin, total protein, triglycerides, and urea nitrogen.

\section{Statistical Analysis}

Data comparisons between autologous and xenogeneic nerve transplant sites, unless otherwise stated, are expressed as mean \pm SEM per transplant type. Elecrtrophysiology statistical comparisons were performed as one-way analysis of variance tests with the Student-Newman-Keuls multiple comparisons method. Immunoglobulin analysis was performed as an unpaired t-Test using Prism Graph Pad version 9.1.0 software (Prism, San Diego, CA USA). P values less than 0.05 were considered statistically significant for all analysis. 


\section{References}

1. Zilic, L. et al. An anatomical study of porcine peripheral nerve and its potential use in nerve tissue engineering. J Anat 227, 302-314. ISSN: 0021-8782. https://www.ncbi.nlm. nih.gov/pmc/articles/PMC4560565/ (2019) (Sept. 2015).

2. Grinsell, D. \& Keating, C. P. Peripheral nerve reconstruction after injury: a review of clinical and experimental therapies. Biomed Res Int 2014, 698256. ISSN: 2314-6141 (2014).

3. Bittner, G. D., Schallert, T. \& Peduzzi, J. D. Degeneration, Trophic Interactions, and Repair of Severed Axons: A Reconsideration of Some Common Assumptions. Neuroscientist 6. Publisher: SAGE Publications Inc STM, 88-109. ISSN: 1073-8584. https://doi.org/10.1177/107385840000600207 (2021) (Apr. 1, 2000).

4. Wagstaff, L. J. et al. Failures of nerve regeneration caused by aging or chronic denervation are rescued by restoring Schwann cell c-Jun. Elife 10, e62232. ISSN: 2050-084X (Jan. 21, 2021).

5. Lanier, S. T., Jordan, S. W., Ko, J. H. \& Dumanian, G. A. Targeted Muscle Reinnervation as a Solution for Nerve Pain. Plastic and Reconstructive Surgery 146, 651e. ISSN: 00321052. https://journals.lww.com/plasreconsurg/Fulltext/2020/11000/Targeted_Muscle_ Reinnervation_as_a_Solution_for.48.aspx (2021) (Nov. 2020).

6. Vasileiadis, A. Bridging of Peripheral Nerve Defects by Autologous Nerve Grafting Personal Experience. MOJ Orthop Rheumatol 5. Publisher: MedCrave Publishing. ISSN: 2374-6939. https://medcraveonline.com/MOJOR/MOJOR-05-00183.pdf (2021) (Aug. 9, 2016).

7. Lee, S. K. \& Wolfe, S. W. Peripheral Nerve Injury and Repair. JAAOS - Journal of the American Academy of Orthopaedic Surgeons 8, 243-252. ISSN: 1067-151X. https://journals.lww.com/jaaos/Fulltext/2000/07000/Peripheral_Nerve_Injury_and_ Repair.5.aspx (2021) (Aug. 2000).

8. Griffin, J. W., Hogan, M. V., Chhabra, A. B. \& Deal, D. N. Peripheral Nerve Repair and Reconstruction. Journal of bone and joint surgery. American volume 95. Place: United States Publisher: Copyright by The Journal of Bone and Joint Surgery, Incorporated, 2144-2151. ISSN: 0021-9355 (2013).

9. Konofaos, P. \& Halen, J. P. V. Nerve Repair by Means of Tubulization: Past, Present, Future. J Reconstr Microsurg 29. Publisher: Thieme Medical Publishers, 149-164. ISSN: 0743-684X, 1098-8947. http://www .thieme- connect.de/DOI/DOI?10.1055/s-0032 1333316 (2021) (Mar. 2013).

10. Althagafi, A. \& Nadi, M. in StatPearls (StatPearls Publishing, Treasure Island (FL), 2021). http://www.ncbi.nlm.nih.gov/books/NBK549848/ (2021).

11. Biomere. PSK18-01 Safety and Immunogenicity Evaluation of Porcine Xenotransplant Skin Grafts in Non-human Primates (Biomere, 2018), 1-17.

12. XenoTherapeutics, Inc. An Open-label Phase 1 Study to Evaluate the Safety and Tolerability of Xeno-Skin ${ }^{\mathrm{TM}}$ for Temporary Coverage of Severe and Extensive, Deep Partial and Full Thickness Burn Wounds Clinical trial registration NCT03695939. submitted: September 28, 2018 (clinicaltrials.gov, Aug. 28, 2020). https://clinicaltrials.gov/ct2/show/NCT03695939 (2021).

13. Chunasuwankul, R. et al. Low dose discontinued FK506 treatment enhances peripheral nerve regeneration. Int Surg 87, 274-278. ISSN: 0020-8868 (Dec. 2002).

14. Wang, L. H. \& Weiss, M. D. Anatomical, Clinical, and Electrodiagnostic Features of Radial Neuropathies. Physical Medicine and Rehabilitation Clinics of North America. 
The Electrodiagnosis of Neuromuscular Disorders 24, 33-47. ISSN: 1047-9651. https://www.sciencedirect.com/science/article/pii/S1047965112000903 (2021) (Feb. 1, 2013).

15. Holzer, P. W. et al. Immunological response in cynomolgus macaques to porcine $\alpha-1,3$ galactosyltransferase knockout viable skin xenotransplants-A pre-clinical study. Xenotransplantation n/a. eprint: https://onlinelibrary.wiley.com/doi/pdf/10.1111/xen.12632, e12632. ISSN: 1399-3089. https://onlinelibrary.wiley.com/doi/abs/10.1111/xen.12632 (2020) (n/a Aug. 11, 2020).

16. Holzer, P. W. et al. A Comparative Examination of the Clinical Outcome and Histological Appearance of Cryopreserved and Fresh Split-Thickness Skin Grafts. J Burn Care Res 38, e55-e61. ISSN: 1559-0488 (Feb. 2017).

17. Biomere. PRT18-01 Neurography Audited Report (Nov. 18, 2019).

18. Yang, R. K. et al. Dose-Dependent Effects of FK506 on Neuroregeneration in a Rat Model: Plastic and Reconstructive Surgery 112, 1832-1840. ISSN: 0032-1052. http://journals. lww.com/00006534-200312000-00012 (2020) (Dec. 2003).

19. Yan, Y., Sun, H. H., Hunter, D. A., Mackinnon, S. E. \& Johnson, P. J. Efficacy of ShortTerm FK506 Administration on Accelerating Nerve Regeneration. Neurorehabil Neural Repair 26. Publisher: SAGE Publications Inc STM, 570-580. ISSN: 1545-9683. https: //doi.org/10.1177/1545968311431965 (2021) (July 1, 2012).

20. Pathology, A. PRT18-01 - Report on Pathology - Audited (Feb. 14, 2020).

21. Chen, Y. et al. Reference values of clinical chemistry and hematology parameters in rhesus monkeys (Macaca mulatta). Xenotransplantation $16 . \quad$ eprint: https://onlinelibrary.wiley.com/doi/pdf/10.1111/j.1399-3089.2009.00554.x, 496-501. ISSN: https://onlinelibrary. wiley.com/doi/abs/10.1111/j.1399-3089.2009.00554.x (2021) (2009).

22. Koo, B.-S. et al. Reference values of hematological and biochemical parameters in young-adult cynomolgus monkey (Macaca fascicularis) and rhesus monkey (Macaca mulatta) anesthetized with ketamine hydrochloride. Laboratory Animal Research 35, 7. ISSN: 2233-7660. https://doi.org/10.1186/s42826-019-0006-0 (2021) (July 24, 2019).

23. Bombeiro, A. L., Lima, B. H. d. M., Bonfanti, A. P. \& Oliveira, A. L. R. d. Improved mouse sciatic nerve regeneration following lymphocyte cell therapy. Molecular Immunology 121, 81-91. https://www.sciencedirect.com/science/article/pii/S0161589019308417 (2021) (May 1, 2020).

24. Roberton, V. H. in Peripheral Nerve Tissue Engineering and Regeneration (eds Phillips, J., Hercher, D. \& Hausner, T.) 1-30 (Springer International Publishing, Cham, 2020). ISBN: 978-3-030-06217-0. https://doi.org/10.1007/978-3-030-06217-0_15-1 (2021).

25. Denner, J. Detection of cell-free pig DNA using integrated PERV sequences to monitor xenotransplant tissue damage and rejection. Xenotransplantation 28. eprint: https://onlinelibrary.wiley.com/doi/pdf/10.1111/xen.12688, e12688. ISSN: 1399-3089. https://onlinelibrary.wiley.com/doi/abs/10.1111/xen.12688 (2021) (2021).

26. Denner, J. Why was PERV not transmitted during preclinical and clinical xenotransplantation trials and after inoculation of animals? Retrovirology 15, 28. ISSN: 1742-4690.

https://retrovirology.biomedcentral.com/articles/10.1186/s12977-018-0411-8 (2019) (Dec. 2018). 
27. Holzer, P. et al. Vital, Porcine, Gal-Knockout Skin Transplants Provide Efficacious Temporary Closure of Full-Thickness Wounds: Good Laboratory Practice-Compliant Studies in Nonhuman Primates. J Burn Care Res 41, 229-240. ISSN: 1559-0488 (2020).

28. Arora, S., Goel, N., Cheema, G. S., Batra, S. \& Maini, L. A Method to Localize The Radial Nerve Using the 'Apex Of Triceps Aponeurosis' as a Landmark. Clin Orthop Relat Res 469, 2638-2644. ISSN: 0009-921X. https : // www . ncbi .nlm . nih.gov/pmc/articles / PMC3148375/ (2021) (Sept. 2011).

29. Wang, D. et al. A simple model of radial nerve injury in the rhesus monkey to evaluate peripheral nerve repair. Neural Regeneration Research 9, 1041. ISSN: 1673-5374. http: //www.nrronline.org/text.asp?2014/9/10/1041/133166 (2018) (2014).

30. Injury of Radial Nerve: Causes, Symptoms \& Diagnosis Healthline. https://www.healthline.com/health/radial-nerve-dysfunction (2021).

31. Gragossian, A. \& Varacallo, M. in StatPearls (StatPearls Publishing, Treasure Island (FL), 2021). http://www.ncbi.nlm.nih.gov/books/NBK537304/ (2021).

32. Mohanna, P. N., Young, R. C., Wiberg, M. \& Terenghi, G. A composite poly-hydroxybutyrate-glial growth factor conduit for long nerve gap repairs. J Anat 203, 553-565. ISSN: 0021-8782 (Dec. 2003).

33. Scobie, L. et al. Long-Term IgG Response to Porcine Neu5Gc Antigens without Transmission of PERV in Burn Patients Treated with Porcine Skin Xenografts. The Journal of Immunology 191, 2907-2915. ISSN: 0022-1767, 1550-6606. http://www.jimmunol.org/cgi/doi/10.4049/jimmunol.1301195 (2018) (Sept. 15, 2013).

34. Tripathi, V. \& Obermann, W. M. J. A Primate Specific Extra Domain in the Molecular Chaperone Hsp90. PLOS ONE 8. Publisher: Public Library of Science, e71856. ISSN: 1932-6203. https://journals.plos.org/plosone/article? id=10.1371/journal.pone.0071856 (2021) (Aug. 9, 2013). 


\section{Acknowledgements}

The research reported in this publication was supported in part by the Department of Defense under Award Number W18XWH-17-I-0454. We thank Dana Barberio of Edge Bioscience Communications for her writing and editorial assistance on a previous version of this manuscript. We thank the animal care staff at Biomere for their assistance with this GLP study. The schematic figures were created with BioRender.com.

\section{Author Contributions}

P.H., C.L.C., and R.M. obtained the funding and designed the experiments. P.H. and C.L.C. performed all animal surgeries. A.L. provided the animal facilities, resources, veterinary observations, and performed behavioral experiments, hematology, and clinical chemistry. M.M. performed electrophysiology. L.S., J.T., and N.G. performed PERV analysis and all PCR. J.W. performed all pathology. B.O. and A.L. performed immunogenicity assays. P.H., E.J.C., D.L., M.M., J.W., B.O., L.S., and R.M. performed data analysis. D.L. and E.J.C. drafted the manuscript. S.G., J.D., and all authors revised the manuscript for critical content. P.H., K.R., and J.A. managed all administrative, technical, or supervisory tasks.

\section{Competing Interests}

C.L.C. previously served as a board member of XenoTherapeutics, Inc, 501(c)3. L.S. is a member of the safety review board of XenoTherapeutics, Inc, 501(c)3. P.H., J.A., and C.L.C. are cofounders of XenoTherapeutics, Inc, 501(c)3. P.H., J.A., K.R., E.J.C., and R.M. are inventors on patent applications related to this work. P.H., E.J.C., D.L., K.R., J.A., and R.M., received financial compensation as employees of XenoTherapeutics, Inc, 501(c)3. Biomere, StageBio, and XenoDiagnositcs are paid vendors for XenoTherapeutics, Inc, 501(c)3. The other authors declare no competing interests.

\section{Materials and Correspondence}

Correspondence and requests for materials should be addressed to P.H. 